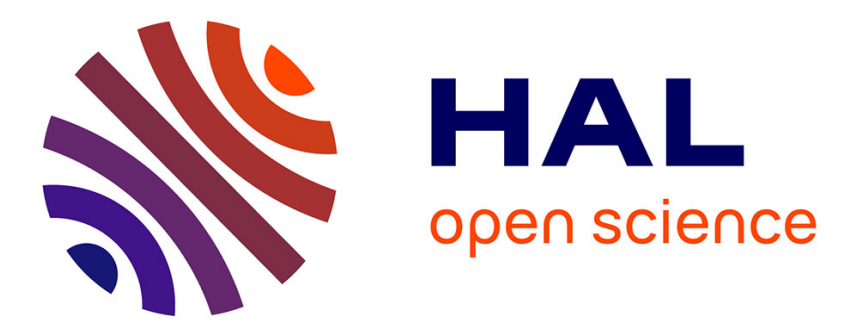

\title{
How to Get an Efficient yet Verified Arbitrary-Precision Integer Library
}

Raphaël Rieu-Helft, Claude Marché, Guillaume Melquiond

\section{To cite this version:}

Raphaël Rieu-Helft, Claude Marché, Guillaume Melquiond. How to Get an Efficient yet Verified Arbitrary-Precision Integer Library. 9th Working Conference on Verified Software: Theories, Tools, and Experiments, Jul 2017, Heidelberg, Germany. pp.84-101, 10.1007/978-3-319-72308-2_6 . hal$01519732 \mathrm{v} 2$

\section{HAL Id: hal-01519732 \\ https://hal.inria.fr/hal-01519732v2}

Submitted on 31 Aug 2017

HAL is a multi-disciplinary open access archive for the deposit and dissemination of scientific research documents, whether they are published or not. The documents may come from teaching and research institutions in France or abroad, or from public or private research centers.
L'archive ouverte pluridisciplinaire HAL, est destinée au dépôt et à la diffusion de documents scientifiques de niveau recherche, publiés ou non, émanant des établissements d'enseignement et de recherche français ou étrangers, des laboratoires publics ou privés. 


\title{
How to Get an Efficient yet Verified Arbitrary-Precision Integer Library
}

\author{
Raphaël Rieu-Helft ${ }^{1,2,3}$, Claude Marché2,3, and Guillaume Melquiond ${ }^{2,3}$ \\ 1 École Normale Supérieure, F-75230 Paris \\ 2 Inria, Université Paris-Saclay, F-91120 Palaiseau \\ 3 LRI (CNRS \& Univ. Paris-Sud), Université Paris-Saclay, F-91405 Orsay
}

\begin{abstract}
The GNU Multi-Precision library is a widely used, safetycritical, library for arbitrary-precision arithmetic. Its source code is written in $\mathrm{C}$ and assembly, and includes intricate state-of-the-art algorithms for the sake of high performance. Formally verifying the functional behavior of such highly optimized code, not designed with verification in mind, is challenging. We present a fully verified library designed using the Why3 program verifier. The use of a dedicated memory model makes it possible to have the Why3 code be very similar to the original GMP code. This library is extracted to $\mathrm{C}$ and is compatible and performancecompetitive with GMP.
\end{abstract}

Keywords: arbitrary-precision arithmetic, deductive program verification, C language, Why3 program verifier

\section{Introduction}

The GNU Multi-Precision library $4_{4}^{4}$ GMP for short, is a widely used library for arithmetic on integers and rational numbers of arbitrary size. Its applications range from academic research (e.g. research on computational algebra) to concrete applications of our daily life (e.g. security of Internet applications). Some of these applications make GMP safety-critical. In this paper, we focus on the mpn component of GMP, which is dedicated to non-negative integers and is used as a basis in all others components. For maximal performance, GMP uses numerous state-of-the-art algorithms for basic operations like addition, multiplication, and division; these algorithms are selected depending on size of the numbers involved. Moreover, the implementation is written in low-level $\mathrm{C}$ code, and depending on the target computer architecture, some parts are even rewritten in assembly.

Being highly optimized for run-time efficiency, the code of GMP is intricate and thus error-prone. It is extensively tested but it is hard to reach a satisfactory coverage in practice: the number of possible inputs is very large, the different branches of the algorithms are numerous, and some of them are taken with a very low probability (some branches are taken with probability $2^{-64}$ or less).

\footnotetext{
4 http://gmplib.org/
} 
Bugs in the division, occurring with very low probability, were discovered in the past 5 Verifying the code for all inputs using static program verification is thus desirable. Such a verification, however, is difficult, not only because of the intrinsic complexity of the algorithms, but also because the code is written in a low-level language with performance in mind, but not verification. In this paper we present an approach to address this latter challenge.

The main idea of our approach is to first write the code in some higherlevel language, namely the programming language WhyML supported by the Why3 verification environment. This language is designed for static verification with respect to some functional behavior specified using an expressive formal specification language. The main issue is then to convert such a high-level code into an efficient executable code. Our approach is to first design a dedicated memory model in Why3, on top of which we then implement our functions. This memory model is designed to permit a direct compilation from WhyML to C. As a result, we obtain the first fully verified library, compatible with GMP (function signatures are the same), and almost as efficient as GMP on medium-sized integers (up to around 20 words of 64 bits). The full development is available from http://toccata.lri.fr/gallery/multiprecision.en.html.

The paper is organized as follows. In Section 2, we present the design of our dedicated memory model and explain how it is suitable for compilation to C. In Section 3, we present the specifications and the algorithms we implemented for arithmetic operations. In Section 4 we present an extensive evaluation of the efficiency of the generated code, comparing it with GMP. We discuss related work in Section 5 and we conclude in Section 6.

\section{From WhyML to $\mathrm{C}$}

Why3 is an environment for deductive program verification, providing a rich language for specification and programming, called WhyML. WhyML is used as an intermediate language for verification of C, Java, and Ada programs [12]18, and is also intended to be comfortable as a primary programming language [13]. WhyML function definitions are annotated with pre- and postconditions both for normal and exceptional termination, and loops are annotated with invariants.

The specification component of WhyML [519, used to write program annotations and background theories, is an extension of first-order logic. It features ML-style polymorphic types (prenex polymorphism), algebraic data types, inductive and co-inductive predicates, and recursive definitions over algebraic types. Constructions like pattern matching, let-binding, and conditionals, can be used directly inside formulas and terms. Why3 comes with a rich standard library providing general-purpose theories useful for specifying programs, including integer and real arithmetic. From programs annotated with specifications, Why3 generates proof obligations and dispatches them to multiple provers, including SMT solvers Alt-Ergo, CVC4, Z3, TPTP first-order provers E, SPASS, Vampire,

\footnotetext{
${ }^{5}$ Look for 'division' at https://gmplib.org/gmp5.0.html
} 


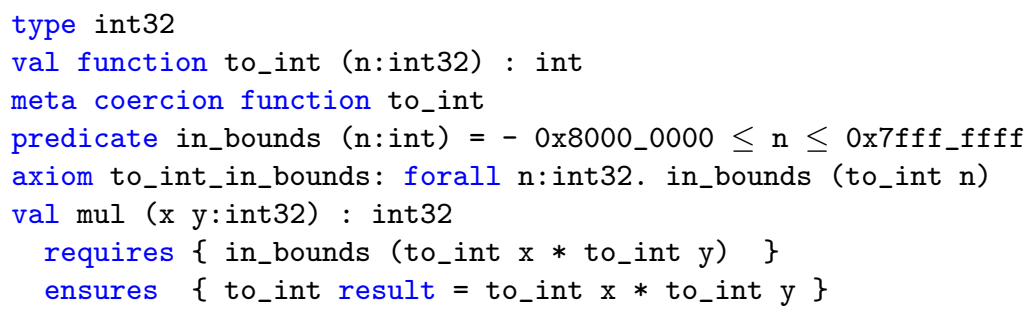

Fig. 1: Excerpt from the specification of 32-bit machine words in Why3.

and interactive theorem provers Coq, Isabelle, and PVS. As most of the provers do not support some of the language features, typically pattern matching, polymorphic types, or recursion, Why3 applies a series of encoding transformations to eliminate unsupported constructions before dispatching a proof obligation.

The programming part of WhyML is a dialect of ML with a number of restrictions to make automated proving easier. The major restriction concerns the potential aliasing of mutable data structures. The language and its typing system are designed so that all aliases are statically known. Technically, the typing system computes read and write effects on singleton regions for each sub-expression [10]. These effects allow the design of a weakest precondition calculus that is as simple as for the while languages usually considered in classical Hoare logic. Verification of complex code with Why3 and automatic provers typically expects user guidance through addition of intermediate assertions [19] and verification-only code (ghost code) [11. See Why3's Web site $6^{6}$ for an extensive tutorial and a large collection of examples [6].

The extraction mechanism of Why3 amounts to compiling WhyML code into a regular programming language while forgetting verification-only annotations. Why3 natively supports extraction to OCaml. For our work we had to implement extraction to $\mathrm{C}$ code. To obtain $\mathrm{C}$ code that includes low-level memory access through pointers, it was mandatory to start by designing a Why3 model of the $\mathrm{C}$ memory heap and pointers, where potential pointer aliasing is controlled in a way that accommodates WhyML typing system. The description of this memory model and the extraction to $\mathrm{C}$ is the purpose of the rest of this section.

\subsection{Machine words and arithmetic primitives}

In WhyML, only the type int of unbounded mathematical integers is a built-in data type. Machine integers are defined instead in Why3's standard library, specified either in terms of intervals of mathematical integers or with bitvectors 15. We use the first option here, which is roughly described in Fig. 1 for signed 32-bits words. The type int32 is abstract, equipped with a projection to_int mapping words to their mathematical value. Predicate in_bounds together with

\footnotetext{
6 http://why3.lri.fr/
} 


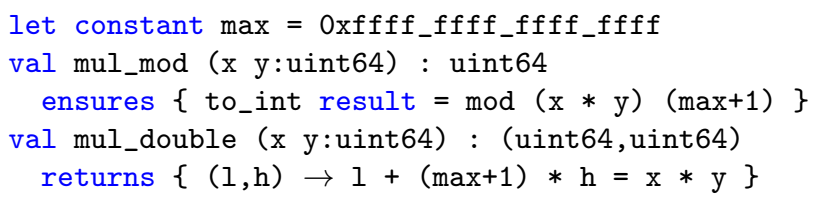

Fig. 2: Multiplication operations on uint64.

axiom to_int_in_bounds specify their possible range. Arithmetic operators like multiplication are then specified in terms of a pre-condition preventing overflows, and a post-condition giving the expected value of the result. Notice the special meta declaration which is a recent addition in Why3. It indicates that int32 words should be implicitly cast to their integer values in specifications. For example, in the contract of function mul, we could omit all occurrences of to_int, which we do in the rest of the paper.

To implement arbitrary-precision arithmetic, we have added primitive operations that allow overflows. This is shown in Fig. 2 for unsigned 64-bit words. The function mul_mod has a wrap-around semantics (result is taken modulo $2^{64}$ ), while the function mul_double returns the full product as a pair of words. Similarly, addition and subtraction come in different flavors (defensive against overflow, 2-complement, with carry in/out). Logical shifts also have both a defensive version and a version with a two-word output. Finally, there is only one division primitive, which takes a two-word numerator and a one-word denominator, and computes a quotient and a remainder.

Regarding extraction, all these data types for machine words are translated into their relevant $\mathrm{C}$ types (e.g. uint64_t). The axiomatized operations are replaced by their equivalent native $\mathrm{C}$ functions when possible. For example, both operations mul and mul_mod are extracted to $\mathrm{C}$ multiplication, since $\mathrm{C}$ operators on unsigned integer types are guaranteed to have the expected semantics for overflows. The mul_double operation, however, does not map to any $\mathrm{C}$ operator, so we import the corresponding operation from GMP's longlong.h file. Reusing GMP's primitives does not only make our library portable to numerous architectures, but it also makes for fairer benchmarks, allowing us to compare the efficiency of big integer algorithms independently of the primitives.

\subsection{A simple model for $\mathrm{C}$ pointers and heap memory}

Arbitrary-precision integers are represented in $\mathrm{C}$ as buffers of unsigned machine words. The functions manipulate pointers, make use of aliasing, and sometimes operate in place. To implement these functions in WhyML, we design a model where the needed pointer operations are axiomatized, as shown in Fig. 3 . At extraction, these operations are then directly replaced by their $\mathrm{C}$ equivalents, indicated as comments in Fig. 3. Our model only specifies the $\mathrm{C}$ features we need. For pointer arithmetic, we only model incrementation of a pointer by an 


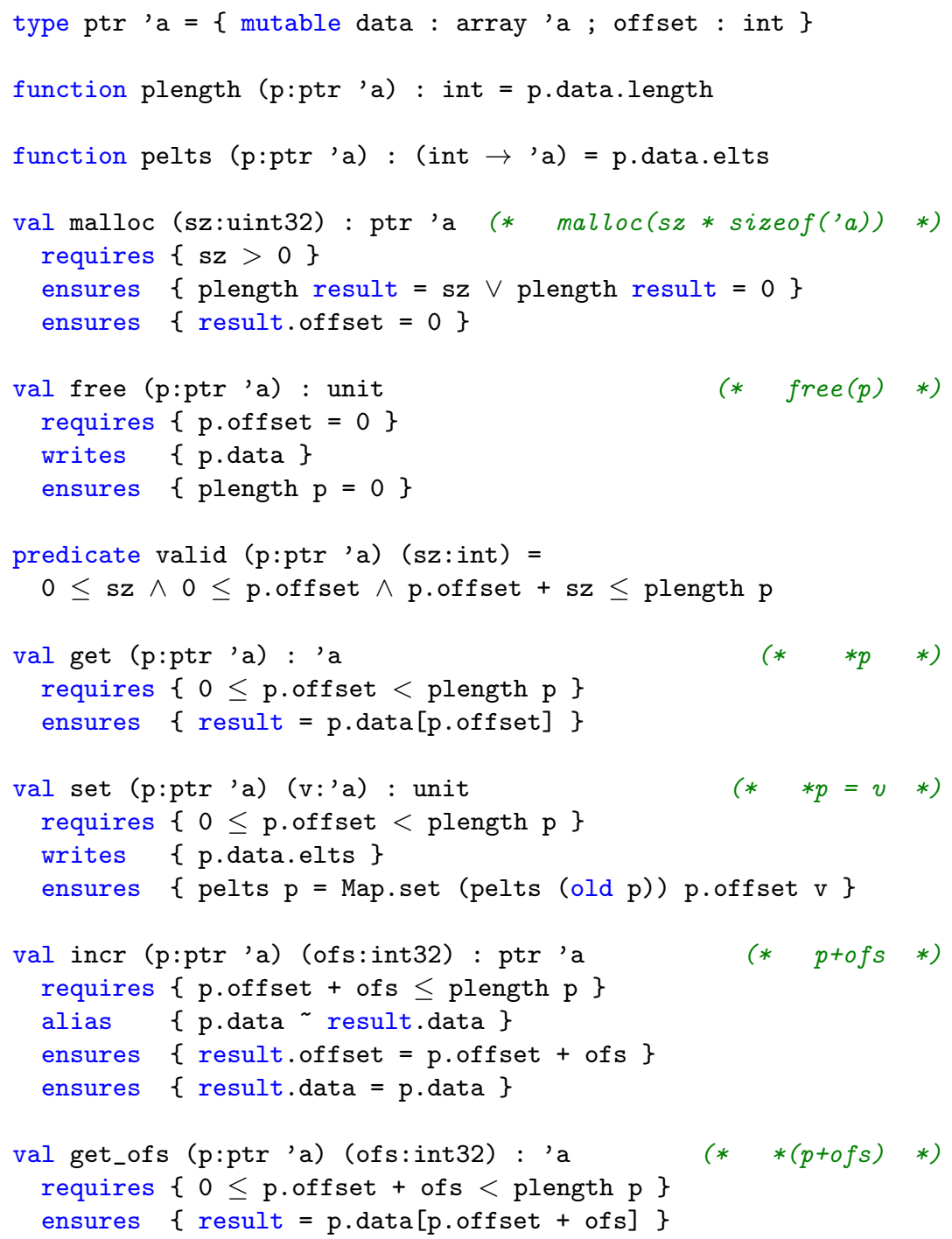

Fig. 3: A Why3 memory model for $\mathrm{C}$ pointers and heap memory.

integer, as we have no use for pointer comparisons or subtractions. We do not need pointer cast either, nor do we need the $\mathrm{C}$ address-of operator \&. Generally speaking, we do not use a model that would cover all features of $\mathrm{C}$, because we want to benefit from the non-aliasing properties provided by Why3's static typing system. The benefit is that both the specifications and the proofs are simpler. With a general model of $\mathrm{C}$ heap memory, we would need to state a lot of non-aliasing hypotheses among the pointers, these properties would generate 
extra VCs to be established by back-end provers, moreover the other VCs will be more difficult to discharge.

The $\mathrm{C}$ heap memory is seen as a set of memory blocks called objects in the C99 standard. The WhyML polymorphic type ptr 'a (Fig. 3. line 1) represents pointers to blocks storing data of type 'a. The field data of a pointer is an array containing the block content, while the field off set indicates which array cell it points to. This construction supports pointer aliasing: several pointers may reference the same array (and thus point inside the same memory block). Thanks to WhyML's region-based type system, an assignment through one pointer is propagated to other pointers.

Pointers are allocated by the malloc function. In case of failure it returns an invalid pointer, represented by a block of length 0 . As such, we forbid passing 0 to malloc. The free function invalidates its parameter by setting the length of its block to 0. A pointer is considered valid for a size $s$ (Fig. 3, line 17) if its offset plus $s$ does not exceed the size of its block. The function get (line 20) represents pointer dereferencing for reading. The function set represents memory assignment; the writes clause specifies the expected write effect on the block.

The incr function (line 29) returns the sum of a pointer and an integer. Just as in the C standard [16, Section 6.5.6, "Additive Operators"], one may only compute a pointer that points inside a valid block or to the element just past it. The Why3 keyword alias in the signature of incr declares the aliasing of the returned pointer with the pointer parameter. Behind the scenes, it unifies the regions of p.data and result.data 10. This aliasing is correct not only with respect to setting the contents of the pointed block, but also with respect to free. This makes it possible to write a particularly short specification for free: the writes effect on p.data induces a so-called reset on it [10], meaning that the region formerly pointed by $\mathrm{p}$ can no longer be accessed by any of its aliases, which are invalidated.

\subsection{Extracting to idiomatic C code}

The main objective of our extraction is to produce code that is correct and as efficient as possible for our arbitrary-precision library. Some WhyML language features, such as algebraic types and higher-order functions, are hard to translate into $\mathrm{C}$ because they would require introducing complex constructions like closures and automatic memory allocation and deallocation. Therefore, we decided to support only a small fragment of the WhyML language in our extraction. The goal is not so much to extract arbitrary WhyML code to $\mathrm{C}$ as to extract imperative, almost C-like WhyML code to a simple and efficient $\mathrm{C}$ program. The supported features of WhyML are those that can be translated straightforwardly to $\mathrm{C}$, such as loops or references. What we gain by giving up on so many language features is that the extraction process is extremely straightforward, and the extracted code resembles the WhyML code line-to-line, with very little added complexity. This makes it easier to obtain efficient $\mathrm{C}$ code, as the WhyML programmer can have a good idea of what the extracted code will be like. The 


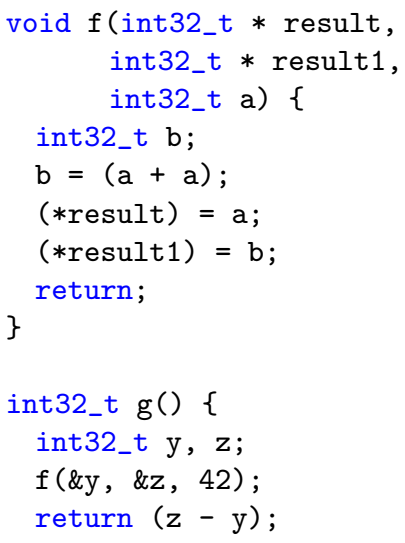

Fig. 4: WhyML function returning a tuple (on the left) and its C extraction.

straightforwardness of the extraction also gives a measure of additional trust in the extracted code and in the extraction process, which is not formally verified.

We now present in more details a few language features that we need to design our library, for which the translation to $\mathrm{C}$ is not direct.

Compilation of exceptions into break or return statements. WhyML does not support certain standard imperative constructs natively. For example, it provides neither break nor return, which are used by some GMP algorithms, e.g. big integer comparison (Section 3.2). So, we encode these constructs using WhyML's exception mechanism. Our extraction recognizes when exceptions can be turned into break or return statements. For break, we essentially detect the following pattern and extract all instances of raise B in the body of the loop (but not inside potential inner nested loops) as break.

try while ... do ... raise $B$... done with $B \rightarrow()$ end

For return, we similarly detect the following pattern of function definitions and extract all instances of raise ( $R$ e) as return e.

let $f(\operatorname{args})=\ldots$; try $\ldots$ raise $(R$ e) $\ldots$ with $R \quad v \rightarrow v$ end

Note that the try with construct must be in tail position of the function body. Our extraction recognizes these patterns independently of the names of the exceptions being used. Any try with or raise construct that does not fit in any of these patterns causes the program to be rejected by our extraction.

Multiple return values. Many of our WhyML functions, particularly arithmetic primitives, return multiple values in a tuple, as can be seen with the mul_double primitive (Fig. 22). This has no native equivalent in C. We choose to 
extract each function returning a tuple as a $\mathrm{C}$ function returning void, taking as extra parameters a pointer per component of the tuple. We detect the call pattern

let $(x 1, x 2, \ldots)=f($ args $)$ in $\ldots$

and extract it as

$\mathrm{f}(\& \mathrm{x} 1, \& \mathrm{x} 2, \ldots, \operatorname{args}) ; \ldots$

Fig. 4 shows the $\mathrm{C}$ program extracted from a WhyML code that defines and calls a function that returns a tuple.

\section{Computing with arbitrary-precision integers}

\subsection{Algorithm specifications}

Just as in GMP, we represent natural integers as buffers of unsigned integers called limbs. We set a radix $\beta$ (generally $\beta=2^{32}$ or $2^{64}$, but the proofs only require it to be a power of 2). Any natural number $N$ has a unique radix- $\beta$ decomposition $\sum_{k=0}^{n-1} a_{k} \beta^{k}$, which is represented as the buffer $a_{0} a_{1} \ldots a_{n-1}$ (with the least significant limb first).

For efficiency, there is no memory management in the low-level functions, so the caller code has to keep track of number sizes. Operands are specified by a pointer to their least significant limb and a limb count of type int32.

type limb = uint64

type $\mathrm{t}=\operatorname{ptr} \mathrm{limb}$

If a pointer $a$ is valid over a size $n$, we denote:

$$
\operatorname{value}(a, n)=\overline{a_{0} \ldots a_{n-1}}=\sum_{k=0}^{n-1} a_{k} \beta^{k} .
$$

In our Why3 development, value is defined recursively

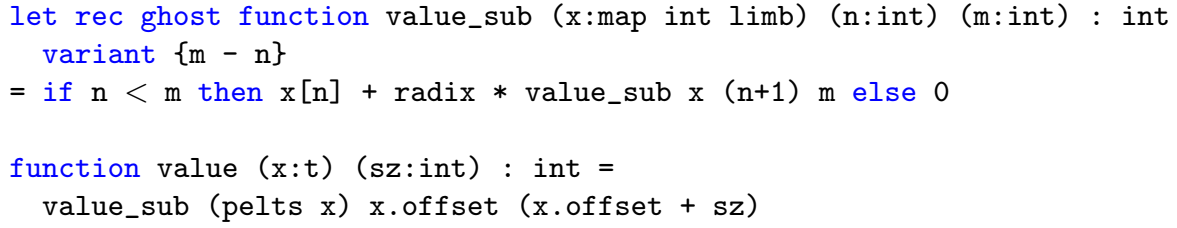

While the functions of our library use only machine types (pointers, limbs, etc), their specifications are expressed in terms of mathematical integers through extensive use of the function value. As an example, Fig. 5 shows the specification of the addition function. Note that the region-based type system forbids aliasing $\mathrm{r}$ with $\mathrm{x}$ or $\mathrm{y}$. Notice also that the specification is well-typed because the conversion functions from int32 and limb to int are coercions: otherwise many applications of to_int would be required. 


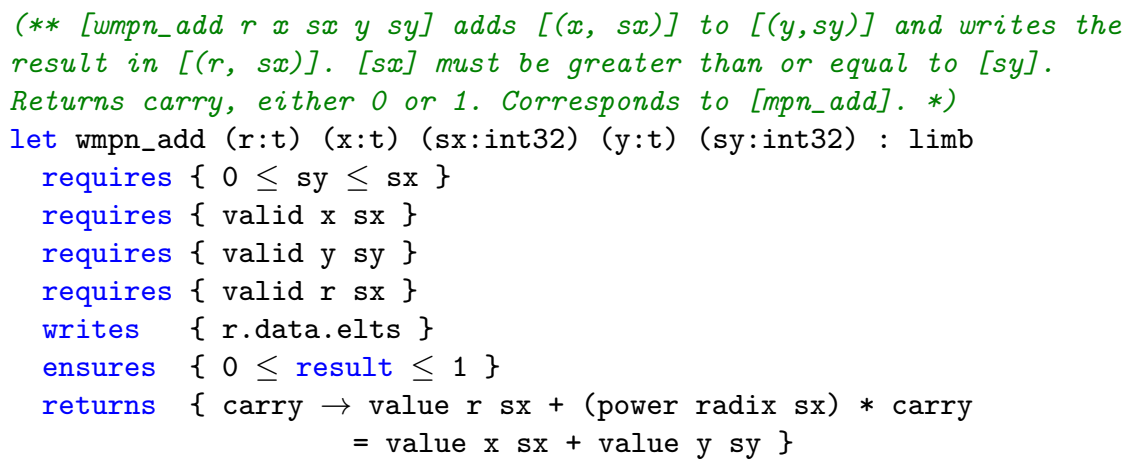

Fig. 5: Specification of wmpn_add.

\subsection{Example of proved algorithm: comparison}

Let us look at the Why3 implementation of GMP's mpn_cmp function, shown in Fig. 6. Just like GMP, this is the only comparison function on natural integers provided by our library. The mpn_cmp function takes two pointers to the integers as arguments, as well as the size of the pointed buffers. It returns $-1,0,1$, depending on the way the numbers are ordered. Our implementation has the same interface and the same behavior. The algorithm is very straightforward: it simply iterates both operands until it finds a difference, starting at the most significant limb. Once a difference is found, we can conclude immediately. If no difference is found, then the integers are equal.

The most important part of the proof is the loop invariant at line 10: both source operands are identical from offsets $i+1$ to $n$. The following lemma is used to prove the postcondition. It simply states that two big integers have the same value if their limbs are equal.

Lemma 1 (value_sub_frame). Let $a_{0}, \ldots, a_{n-1}, b_{0}, \ldots, b_{n-1}$ such that for all $i$, $a_{i}=b_{i}$. Then $\overline{a_{0} \ldots a_{n-1}}=\overline{b_{0} \ldots b_{n-1}}$.

The proof is a straightforward induction, which translates well into a Why3 lemma function where the recursive call provides the induction hypothesis.

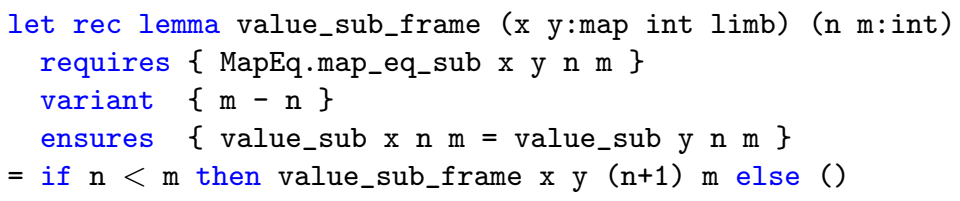

This lemma makes it possible to conclude that the numbers are equal if no difference was found by the end of the loop. Notice that the loop body raises an exception as soon as a difference is found. This emulates the return-inside-a-loop pattern found in imperative languages. At extraction, this pattern is detected and the extracted code simply has a return inside the main loop (Section 2.3). Fig. 7 shows the extracted code for the wmpn_cmp function. 


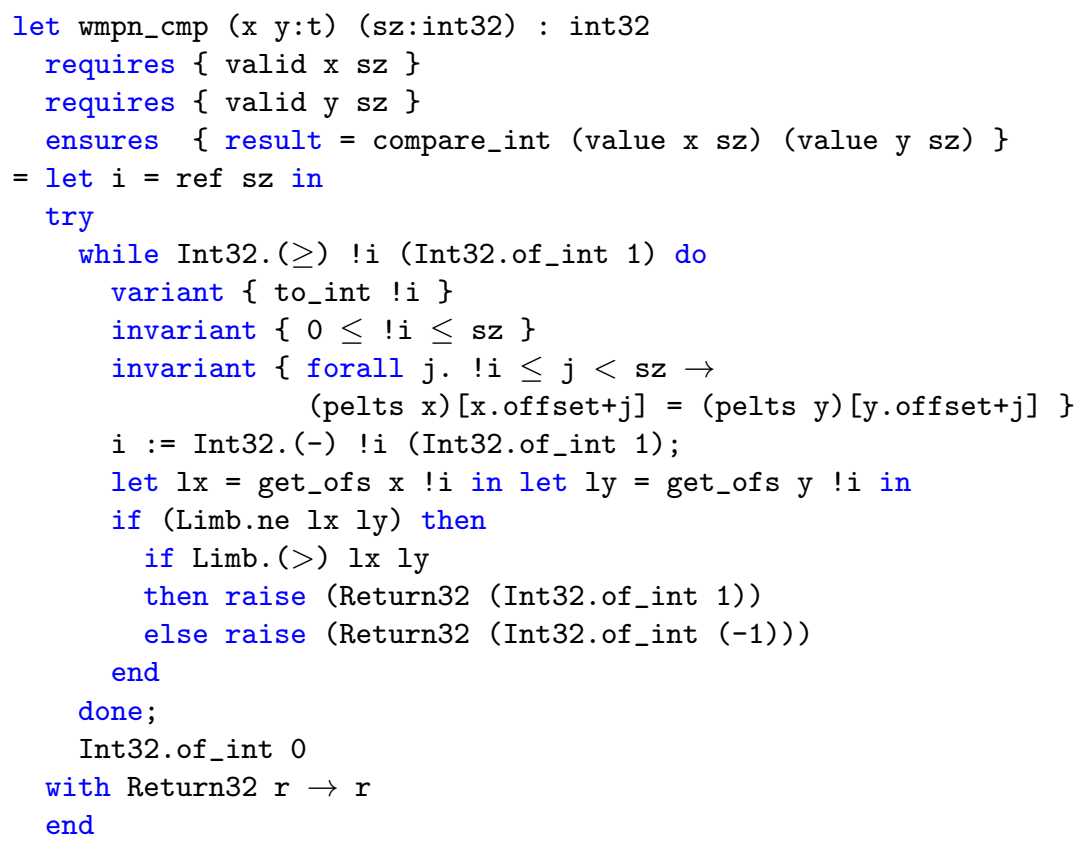

Fig. 6: Why3 implementation of mpn_cmp.

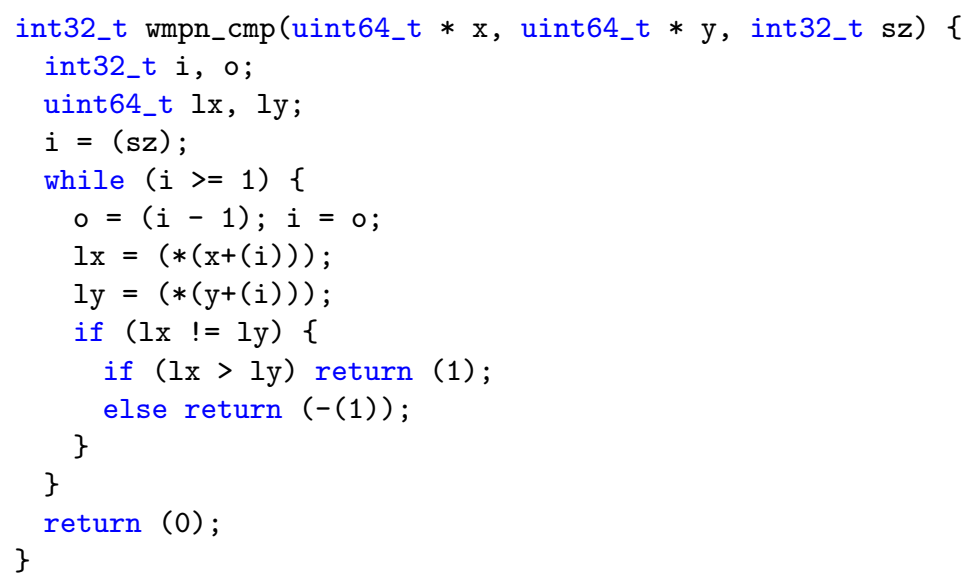

Fig. 7: Extracted C code for wmpn_cmp.

\subsection{Trickier example: long division}

Let us now showcase one of the many algorithmic tricks from GMP that we ported in our implementation. Long division consists in computing the quotient $q$ 


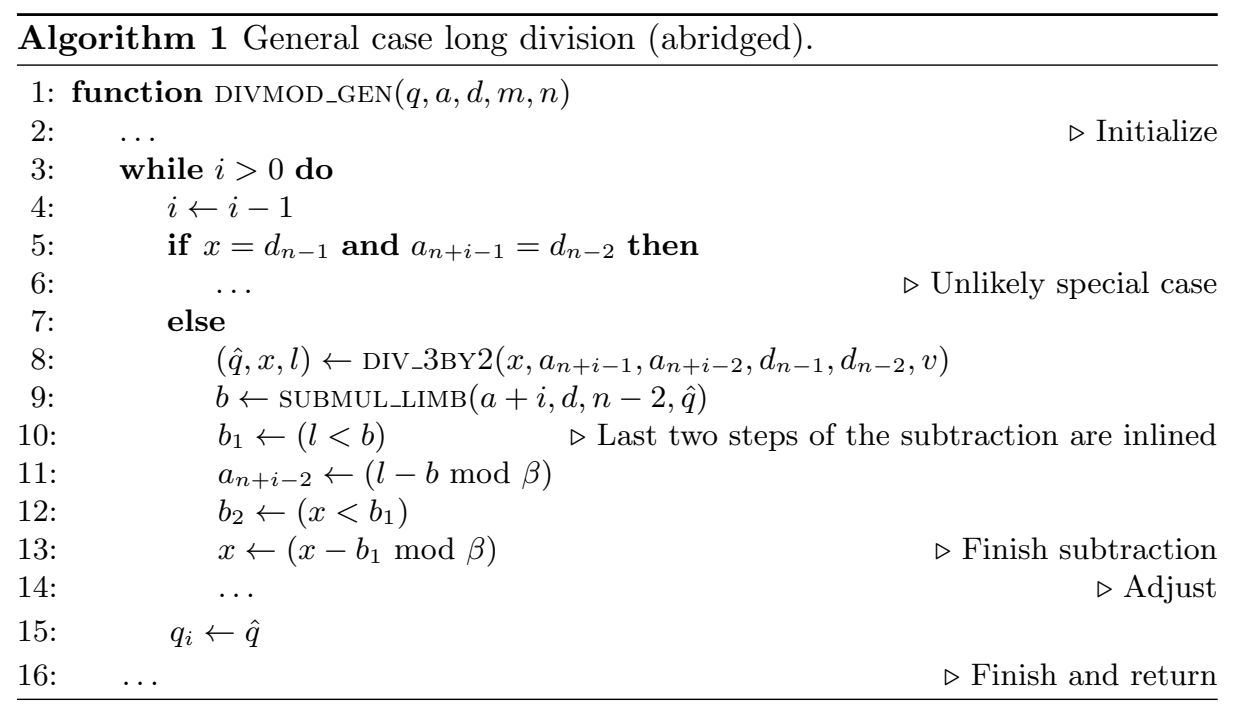

and remainder of the division of a big integer $a$ of size $m$ by a big integer $d$ of size $n$. It is a significantly more complex problem than long addition or multiplication. Algorithm 1 is an excerpt of the general case algorithm for long division in GMP (file mpn/generic/sbpi1_div_qr.c).

The algorithm consists in computing the limbs of the quotient one by one, starting with the most significant one. The numerator $a$ is overwritten at each step to contain the partial remainder. At each iteration of the loop (with $i$ decreasing from $m-n$ ), we compute a quotient limb $\hat{q}$ by dividing the three most significant limbs of the current remainder $a$ (of size $n+i$ ) by the two most significant limbs from the denominator $d$. We then subtract from the high part of the current remainder the product of that quotient limb by the denominator. Note that the most significant limb of the current remainder is never stored back to $a_{n+i}$. It is kept in the local variable $x$ as an optimization.

Let us take a closer look at lines 9 to 13 in Algorithm 11, which expose another optimization of GMP meant to shave a few more processor cycles. The candidate quotient limb $\hat{q}$ is computed at line 8 , and we need to subtract the product of this quotient limb and the denominator from the current remainder. This could be done with only the function call at line 9 by passing $n$ instead of $n-2$ (or rather $n-1$ and inlining the last step on $x$ ), but we can do better and optimize the last two steps by making use of the remainder that was computed at line 8. Indeed, we can show that the last two steps simply consist in propagating the borrow from the previous subtraction, as the result of the 3 most significant limbs of subtraction is known to be $\overline{\ell x 0}=\ell+\beta x$ in the absence of borrow-in (the postcondition of the division is exactly that $\overline{a_{n+i-2} a_{n+i-1} x}=\hat{q} \times \overline{d_{n-2} d_{n-1}}+\overline{\ell x}$. Therefore, all that is left to do is propagate the borrow on $\overline{\ell x 0}$. Hence, lines 11 to 15 are equivalent to computing the subtraction

$$
\overline{a_{i} \ldots a_{n+i-1} x}-\hat{q} \times \overline{d_{0} \ldots d_{n-1}}
$$




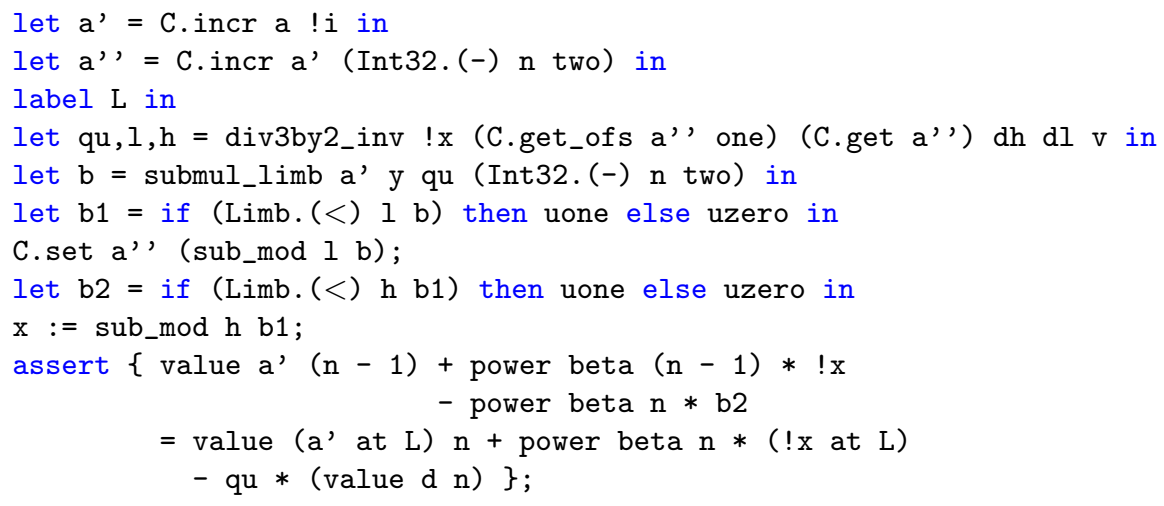

Fig. 8: Transcription (modified for readability) of Algorithm 1, lines 8 to 13.

returning $b_{2}$ as borrow and writing the result in $\overline{a_{i} \ldots a_{n+i-2} x}$ (one limb fewer). This is exactly the last assertion of Fig. 8, which shows an abridged version of our proof for this part of the algorithm.

All in all, this algorithmic trick saves several arithmetic operations: two multiplications, as the two most significant limbs of $d$ are not multiplied by $\hat{q}$, and two subtractions, as in the last two steps, only a carry is propagated instead of doing a subtraction and then propagating a carry. This is far from irrelevant: this loop is the performance-critical one for long integer division, and almost all the cost of the loop is in the submul_limb call (it is the only operation with a cost that scales with the size of the input that is run with non-negligible probability). This trick, which makes the cost of the loop similar to what it would be if the denominator was two limbs shorter, illustrates the kind of GMP implementation details that we have to preserve in order to keep up in terms of performance.

\subsection{Statistics on the proof effort}

We have implemented and verified functions for performing addition, subtraction, multiplication, division, comparison, and logical shifts on arbitrary-precision integers. In many cases, we also provide lower-level functions for the cases when one of the inputs is a single limb or when the two inputs have the same length (equivalent to the functions suffixed by $\_1$ and _n in GMP 7 ).

This totals 6000 lines of Why3, which break down into 1350 lines of code and 4650 lines of specifications and proofs, most of which are assertions. The theorem provers Alt-Ergo, CVC3, CVC4, Eprover, and Z3 are used. All of these provers are necessary for at least some subgoals. It is hard to precisely characterize which subgoals are discharged by each prover, but we can provide some heuristics. Typically, CVC3 is the best of these provers at discharging non-linear

\footnotetext{
7 http://gmplib.org/manual/Low_002dlevel-Functions.html
} 


\begin{tabular}{|l||c|c|c|c|c|c|}
\hline$n n^{m}$ & 5 & 7 & 10 & 13 & 15 & 20 \\
\hline 5 & $0 \%$ & $7 \%$ & $8 \%$ & $6 \%$ & $8 \%$ & $12 \%$ \\
\hline 7 & - & $5 \%$ & $7 \%$ & $8 \%$ & $9 \%$ & $14 \%$ \\
\hline 10 & - & - & $9 \%$ & $7 \%$ & $7 \%$ & $13 \%$ \\
\hline 13 & - & - & - & $9 \%$ & $7 \%$ & $14 \%$ \\
\hline 15 & - & - & - & - & $6 \%$ & $15 \%$ \\
\hline 20 & - & - & - & - & - & $13 \%$ \\
\hline
\end{tabular}

(a) multiplication

\begin{tabular}{|l||c|c|c|c|c|c|}
\hline$n$ & 5 & 7 & 10 & 13 & 15 & 20 \\
\hline 5 & $130 \%$ & $8 \%$ & $25 \%$ & $18 \%$ & $17 \%$ & $16 \%$ \\
\hline 7 & - & $67 \%$ & $3 \%$ & $14 \%$ & $19 \%$ & $14 \%$ \\
\hline 10 & - & - & $61 \%$ & $2 \%$ & $4 \%$ & $12 \%$ \\
\hline 13 & - & - & - & $33 \%$ & $7 \%$ & $3 \%$ \\
\hline 15 & - & - & - & - & $54 \%$ & $5 \%$ \\
\hline 20 & - & - & - & - & - & $40 \%$ \\
\hline
\end{tabular}

(b) division

Fig. 9: Overhead for $m$-by- $n$ operations.

arithmetic subgoals, with Z3 second. Z3 is also good at proving upper bounds and absence of overflows. CVC4 tends to be the best at proving preconditions such as pointer validity. The $\mathrm{E}$ prover is the best at instantiating hypotheses modulo associativity and commutativity. Finally, Alt-Ergo is the best at instantiating complex lemmas and tends to require fewer cut indications. The total proof time is around 20 minutes. For a more detailed breakdown, refer to http://toccata.lri.fr/gallery/multiprecision.en.html. The proof effort is about 5 person-months, most of it being for the division, for a neophyte in computer arithmetic and automated program verification.

\section{Benchmarks}

We have compared the execution time of our extracted code against GMP on randomly generated medium-sized integers, up to 1280 bits. For bigger inputs, the comparison becomes increasingly meaningless since GMP switches to divideand-conquer algorithms which have a better asymptotic complexity. To prevent GMP from using too many architecture-specific optimizations, we have configured GMP with the --disable-assembly flag, so that GMP uses only generic C code. This is true both for the arithmetic primitives (which we share with GMP to focus the benchmarks on the algorithms rather than the primitives) but also for the operations on big numbers. Indeed, on many architectures, GMP uses handwritten assembly functions for most of the performance-critical big number algorithms, with performances out of reach of even very efficient $\mathrm{C}$ code.

We compare the execution times of GMP (without assembly) and our library on three different functions: addition, multiplication, and division. We do separate measures for all valid combinations of lengths of the input operands between 1 and 20. For each of these, we generate a few thousand random inputs and call each function a hundred times on each input, and record the total time.

For multiplication, our library is between 5 and $10 \%$ slower than GMP across all sizes (Fig. 9a). One possible cause for the discrepancy is the use of a different basic block for addition: while we use a primitive that adds two one-limb integers 
and a carry, GMP uses a primitive that adds two two-limb integers. We intend to switch to GMP's primitive in the near future.

For division, the difference in execution times is much more dependent on the length of the inputs, particularly in the difference in length between numerator and denominator (Fig. 9b). When the length of the denominator is less than half the length of the numerator, our algorithm is quite similar to GMP's and runs in about $20 \%$ more time.

The situation changes when the length $n$ of the denominator is more than half the length $m$ of the numerator, that is, more than the length of the quotient. Indeed, GMP no longer applies Algorithm 1 directly on the operands. Instead, the algorithm is called on the $2 q$ most significant limbs of the numerator and $q$ most significant limbs of the denominator, where $q$ is the length of the quotient. This gives an estimated quotient, and a rather involved adjustment step follows. This alternative algorithm is not yet implemented in our library, which simply applies the general algorithm in all cases.

Note that GMP's adjustment step is somewhat expensive in that it requires the allocation of a long integer. Thus, for the small sizes we are considering, the adjustment step seems to dominate the complexity in such a way that the algorithm switch is only worth it when the denominator is almost as long as the numerator. Thus, for $m / 2 \leq n<m-1$, the overhead of our library is below $10 \%$. It then increases drastically when the sizes of the numerator and denominator get very close: for $n=m-1$, our library is around $25 \%$ slower than GMP; for $n=m$, our library is sometimes twice as slow.

We also compared our library with mini-gmp, a minimalistic implementation of the GMP interface in a single $\mathrm{C}$ file that can be found in the main GMP repository. The mini-gmp division does not implement the alternative algorithm either, which makes our division 10 to $20 \%$ slower than it across the board.

\section{Related Work}

In this work, we have obtained our library in three steps: we first write some WhyML code and specification, we then verify that the code satisfies the specification, finally we extract the $\mathrm{C}$ code from the WhyML code. There are numerous other approaches to obtain some verified $\mathrm{C}$ code; let us mention three examples. In the case of the $\mathrm{B}$ method, an abstract specification is progressively refined until it is detailed enough so that some $\mathrm{C}$ code can be extracted from it 1 . In the case of the Frama-C environment, the $\mathrm{C}$ code is written by hand and it is specified using the behavioral specification language ACSL; the verification is then directly performed at the level of the $\mathrm{C}$ code 8 . Finally, in the case of the seL4 microkernel, the $\mathrm{C}$ code is again written by hand, but so is some Haskell code that models it; the verification process then consists of formally proving that this Haskell code both models the $\mathrm{C}$ code and satisfies a specification written in Isabelle/HOL [17.

Let us focus a bit more on the topic of verifying an arbitrary-precision integer library. Bertot et al. verified the GMP's divide-and-conquer algorithm for square 
root [4. It was performed using the Correctness tool which translates a program and its specification into verification conditions for Coq. In that work, the memory is seen as a large array of machine integers, so function specifications have to tell which zones of memory are left unchanged. Other than that, the way the authors implement and specify their algorithm is quite close to the way we do ours; thus, had they wished to, they could easily have extracted it to C.

Myreen and Curello verified a library with a scope similar to the one presented in this paper, although their division algorithm is simpler than GMP's 21]. The implementation, specification, and verification were done using HOL4. An interesting aspect of this work is that the implementation language is some kind of x86-64 pseudo-assembly, so as to effectively produce a low-level verified library. Another interesting point is that it is not the assembly code that is verified but the Hoare triples obtained by decompiling the corresponding machine code. These triples are formally proved to be compatible with the specification of correct algorithms. The memory model is based on separation logic, and the compiler and decompiler are specifically instrumented to preserve the corresponding assertions about integer separation in the generated triples. The library also supports signed integers but their encoding does not match GMP's.

Affeldt verified a binary GCD algorithm and the functions it depends on 2 . Neither multiplications nor divisions are present. The implementation, specification, and verification were done using Coq. This time, the implementation language is a variant of MIPS assembly. An interesting aspect of this work is that, even if the verified algorithm is not GMP's binary GCD, the numbers are encoded using GMP's layout for signed integers, which incurs a pointer indirection. To account for this complexity, the memory model is again based on separation logic.

Further away from GMP, Berghofer verified an Ada library for performing modular exponentiation [3. It was written and specified using the SPARK subset of Ada and the verification conditions were then proved using Isabelle/HOL. The use of Montgomery multiplication makes it slightly more complicated than the binary GCD example from an algorithmic point of view. There is no need for a memory model, since arbitrary-precision integers are represented using plain Ada arrays and SPARK prevents them from being aliased.

Fischer designed a modular exponentiation library developed for $\mathrm{C}$ and verified using Isabelle/HOL [14. Multiplication and division algorithms are naive and use arbitrary-precision integers represented using garbage-collected doublylinked lists of machine integers. Thus, this library is certainly not meant to be efficient. Aliasing issues are solved by using both a Bornat-like memory model 7], so as to automatically distinguish integer words from pointer words, and frame predicates in specifications, so as to declare which heap positions are possibly modified by a function.

Finally, there have also been various efforts to verify specific cryptography primitives and their underlying arithmetic. Zinzindohoué et al. verified an elliptic curve library written in $\mathrm{F}^{*}$ and meant to be extracted to $\mathrm{C}[22$. A peculiarity is that integers are no longer of arbitrary precision; they are represented by fixed- 
size arrays. Moreover, only part of a machine word is used to store a limb; for instance, a 448-bit integer is stored using 8 limbs of 56 bits (out of 64). As a consequence, arithmetic operations on limbs do not have to be modular (which makes them simpler for SMT solvers to reason about) and carry bits do not have to be propagated. Regarding the memory model, function specifications explicitly tell which parts of the heap are modified.

\section{Conclusions}

Our work aims at devising a formally verified C library that provides the same arbitrary-precision arithmetic primitives as GMP. At the time of this paper, we have implemented and verified the following algorithms from GMP: comparison, addition, subtraction, multiplication, and division. For multiplication and division, those are only the algorithms meant to be used with integers of size less than 20 limbs, that is, the so-called schoolbook algorithms. Moreover, in the case of the division, we are lacking an optimized algorithm when the final quotient is short, which means that the version for computing long quotients is always being called (unless the divisor is one or two limbs long).

Thanks to our memory model and the notion of pointer it provides, we were able to write the functions the same way GMP developers did. It also made it easy to implement an extraction mechanism to $\mathrm{C}$ for Why3. Moreover, since this memory model piggybacks on the region mechanism of Why3, we did not have to bother with pointer aliasing, so the specification of the functions is just about their arithmetic properties, contrarily to most of the other verified libraries.

Despite the terminology, the algorithms we have considered are far more intricate than the algorithms one finds in a schoolbook and are still the topic of active research 20. For instance, the division operator is designed to correctly compute the remainder after a single pass with probability almost 1, and thus does not incur a correction step. Our code implements all the algorithmic tricks that can be found in the corresponding functions of GMP, which makes our library competitive with GMP's non-assembly implementation. In fact, the extracted C code is so close to GMP's own code that the formal verification of our library increases the confidence in the correctness of GMP as a by-product.

As it stands, the proof effort for getting a verified GMP-like library is way too costly. Indeed, while the algorithms are highly intricate, the effort required is compounded by the nonlinear nature of the integer properties submitted to the automated solvers. SMT solvers are especially unhelpful there, so the user has to split proofs at a deeper level of detail than what an interactive theorem prover with support for algebraic reasoning would require. Thus, before tackling the implementation and verification of other GMP functions, we intend to work on designing decision procedures dedicated to verifying these arithmetic properties. While the class of nonlinear integer problems is undecidable, the properties that occur when verifying a GMP-like library are sufficiently specific that we have good hope for success. 
Once the issue of proof automation has been tackled, we intend to implement and verify divide-and-conquer algorithms for multiplication (e.g. Toom-Cook algorithms) and division, so as to stay competitive with GMP even for larger integers. We also intend to provide the same high-level interface as GMP for abstract signed arbitrary-precision integers. This comes as a new challenge for the memory model, since most mpz functions allow for aliasing between their arguments. For instance, one can pass the same arbitrary-precision integer as both input and output, so operators have to properly resolve any aliasing issue (e.g. by allocating temporary buffers) before calling into the mpn functions.

Another future work is to extract not only the Why3 code to $\mathrm{C}$, but also the specifications. The $\mathrm{C}$ code could then be verified using an existing $\mathrm{C}$ verification framework, e.g. Frama-C, so that our code extractor no longer needs to be part of the trusted code base. It would be quite costly, however, to translate all the annotations to the ACSL specification language of Frama-C and to perform once again the whole verification, especially since ACSL is not as expressive as Why3. The goal is rather to improve the interaction between Frama-C and Why3 (which Frama-C already uses as a back-end), so as to minimize the proof effort when verifying a $\mathrm{C}$ function whose algorithm has already been proved using Why3.

Acknowledgments. We gratefully thank Pascal Cuoq, Jean-Christophe Filliâtre and Mário Pereira for their comments on preliminary versions of this article.

\section{References}

1. Abrial, J.R.: The B-Book, assigning programs to meaning. Cambridge University Press (1996)

2. Affeldt, R.: On construction of a library of formally verified low-level arithmetic functions. Innovations in Systems and Software Engineering 9(2), 59-77 (2013)

3. Berghofer, S.: Verification of dependable software using SPARK and Isabelle. In: Brauer, J., Roveri, M., Tews, H. (eds.) 6th International Workshop on Systems Software Verification. OpenAccess Series in Informatics (OASIcs), vol. 24, pp. 1531. Dagstuhl, Germany (2012)

4. Bertot, Y., Magaud, N., Zimmermann, P.: A proof of GMP square root. Journal of Automated Reasoning 29(3-4), 225-252 (2002)

5. Bobot, F., Filliâtre, J.C., Marché, C., Paskevich, A.: Why3: Shepherd your herd of provers. In: Boogie 2011: First International Workshop on Intermediate Verification Languages. pp. 53-64. Wrocław, Poland (August 2011), https://hal.inria.fr/ hal-00790310

6. Bobot, F., Filliâtre, J.C., Marché, C., Paskevich, A.: Let's verify this with Why3. International Journal on Software Tools for Technology Transfer (STTT) 17(6), 709-727 (2015), see also http://toccata.lri.fr/gallery/fm2012comp.en.html

7. Bornat, R.: Proving pointer programs in Hoare logic. In: Mathematics of Program Construction. pp. 102-126 (2000)

8. Cuoq, P., Kirchner, F., Kosmatov, N., Prevosto, V., Signoles, J., Yakobowski, B.: Frama-C: A software analysis perspective. In: Proceedings of the 10th International Conference on Software Engineering and Formal Methods. pp. 233-247. No. 7504 in Lecture Notes in Computer Science, Springer (2012) 
9. Filliâtre, J.C.: One logic to use them all. In: 24th International Conference on Automated Deduction (CADE-24). Lecture Notes in Artificial Intelligence, vol. 7898, pp. 1-20. Springer, Lake Placid, USA (June 2013)

10. Filliâtre, J.C., Gondelman, L., Paskevich, A.: A pragmatic type system for deductive verification. Research report, Université Paris Sud (2016), https://hal. archives-ouvertes.fr/hal-01256434v3

11. Filliâtre, J.C., Gondelman, L., Paskevich, A.: The spirit of ghost code. Formal Methods in System Design 48(3), 152-174 (2016)

12. Filliâtre, J.C., Marché, C.: The Why/Krakatoa/Caduceus platform for deductive program verification. In: Damm, W., Hermanns, H. (eds.) 19th International Conference on Computer Aided Verification. Lecture Notes in Computer Science, vol. 4590, pp. 173-177. Springer, Berlin, Germany (Jul 2007)

13. Filliâtre, J.C., Paskevich, A.: Why3 - where programs meet provers. In: Felleisen, M., Gardner, P. (eds.) Proceedings of the 22nd European Symposium on Programming. Lecture Notes in Computer Science, vol. 7792, pp. 125-128. Springer (Mar 2013)

14. Fischer, S.: Formal verification of a big integer library. In: DATE Workshop on Dependable Software Systems (2008), http://www-wjp.cs.uni-sb.de/ publikationen/Fi08DATE.pdf

15. Fumex, C., Dross, C., Gerlach, J., Marché, C.: Specification and proof of high-level functional properties of bit-level programs. In: Rayadurgam, S., Tkachuk, O. (eds.) 8th NASA Formal Methods Symposium. Lecture Notes in Computer Science, vol. 9690, pp. 291-306. Springer, Minneapolis, MN, USA (Jun 2016)

16. International Organization for Standardization: ISO/IEC 9899:1999: Programming Languages - C (2000)

17. Klein, G., Andronick, J., Elphinstone, K., Heiser, G., Cock, D., Derrin, P., Elkaduwe, D., Engelhardt, K., Kolanski, R., Norrish, M., Sewell, T., Tuch, H., Winwood, S.: seL4: Formal verification of an OS kernel. Communications of the ACM 53(6), 107-115 (Jun 2010)

18. Kosmatov, N., Marché, C., Moy, Y., Signoles, J.: Static versus dynamic verification in Why3, Frama-C and SPARK 2014. In: Margaria, T., Steffen, B. (eds.) 7th International Symposium on Leveraging Applications of Formal Methods, Verification and Validation (ISoLA). Lecture Notes in Computer Science, vol. 9952, pp. 461-478. Springer, Corfu, Greece (Oct 2016)

19. Leino, K.R.M., Moskal, M.: Usable auto-active verification. In: Usable Verification Workshop. Redmond, WA, USA (Nov 2010), http://fm.csl.sri.com/UV10/

20. Moller, N., Granlund, T.: Improved division by invariant integers. IEEE Transactions on Computers 60(2), 165-175 (2011)

21. Myreen, M.O., Curello, G.: Proof pearl: A verified bignum implementation in x8664 machine code. In: Gonthier, G., Norrish, M. (eds.) 3rd International Conference on Certified Programs and Proofs (CPP). Lecture Notes in Computer Science, vol. 8307, pp. 66-81. Springer, Melbourne, Australia (Dec 2013)

22. Zinzindohoué, J.K., Bartzia, E.I., Bhargavan, K.: A verified extensible library of elliptic curves. In: Hicks, M., Köpf, B. (eds.) 29th IEEE Computer Security Foundations Symposium (CSF). pp. 296-309. Lisbon, Portugal (Jun 2016) 